Revue européenne des sciences sociales

European Journal of Social Sciences

XLI-125 | 2003

Index de la Revue européenne des sciences sociales

(1995-2002)

\title{
Sur la version colinsienne de l'argument ontologique
}

Ivo Rens

\section{(2) OpenEdition}

Journals

Édition électronique

URL : http://journals.openedition.org/ress/564

DOI : $10.4000 /$ ress. 564

ISSN : 1663-4446

Éditeur

Librairie Droz

Édition imprimée

Date de publication : 1 janvier 2003

Pagination : 143-151

ISBN : 2-600-00844-6

ISSN : 0048-8046

Référence électronique

Ivo Rens, «Sur la version colinsienne de l'argument ontologique », Revue européenne des sciences sociales [En ligne], XLI-125 | 2003, mis en ligne le 01 décembre 2009, consulté le 03 mai 2019. URL http://journals.openedition.org/ress/564 ; DOI : 10.4000/ress.564 


\section{Ivo RENS}

\section{SUR LA VERSION COLINSIENNE DE L'ARGUMENT ONTOLOGIQUE}

Depuis sa réfutation par Kant, l'argument ontologique, c'est-à-dire la preuve rationnelle de l'existence de Dieu à partir de l'essence, n'intéresse plus guère que les historiens. De fait, il tient une place importante dans l'histoire de la philosophie. Enoncé par Anselme de Canterbury (né à Aoste en 1033-1109)ํㅜㄹ contesté par Thomas d'Aquin (1225-1274), puis reformulé successivement par Descartes (1596-1650) et par Leibniz (1646-1716), il illustre magnifiquement ce que Jeanne Hersch a appelé «l'illusion philosophique $»^{2}$.

Toutefois il eût été étonnant que le rationalisme du XIX $\mathrm{X}^{\mathrm{e}}$ siècle se désintéressât de cette problématique. Mais c'est dans un contexte intellectuel totalement renouvelé qu'il apparaît sous la plume d'un philosophe socialiste, le baron Colins de Ham (1783-1859), dans le tome V de sa monumentale Science sociale paru en $1857^{3}$. Ancien officier de la Grande Armée et de la Vieille Garde de Napoléon, cet agronome de formation, qui avait été une douzaine d'années planteur à Cuba et qui de 1835 à 1845 avait entrepris de suivre les cours de la plupart des institutions savantes de Paris, fut l'un des représentants les plus téméraires du siècle de toutes les audaces ${ }^{4}$.

\section{PRÉSUPPOSÉ SCIENTIFIQUE DE COLINS}

L'une des plus grandes controverses scientifiques du XIX ${ }^{\mathrm{e}}$ siècle opposa les partisans du caractère fixe des espèces biologiques, à partir de la Création, aux tenants du transformisme qui postulaient l'évolution des formes du vivant. JeanBaptiste Lamarck (1744-1829) fut le premier naturaliste à soutenir un transformisme scientifique généralisé à toutes les espèces. Il le fit publiquement dès 1800 dans un Discours d'ouverture du 21 floréal de l'an VIII (11 mai 1800), puis il

\footnotetext{
C'est toutefois le moine allemand Bruun, dit Candide de Fulda, qui passe pour être le premier à avoir donné au moyen âge la preuve dialectiquement développée de l'existence de Dieu. Cf. Etienne Gilson, La philosophie au moyen âge, Payot, Paris, 1962 (éd. or. 1944), p. 198.

2 Jeanne Hersch, L'illusion philosophique, Préface de Karl Jaspers, Plon, Paris, 1964.

3 Hippolyte Colins, Science sociale, tome V, p. 1 à 260 et plus particulièrement p. 171 à 260. Cf. aussi Sciene sociale, tome XVI, p. 407 à 427, De la justice dans la science, hors l'Eglise et hors la Révolution, tome II p. 627 - 644, tome III, p. 365 à 368.

4 Sur la vie de Colins, cf. notre Introduction au socialisme rationnel de Colins, A la Baconnière, Neuchâtel, 1968.
} 
développa ses vues dans sa Philosophie zoologique parue en $1809^{5}$. Les autres transformistes français les plus éminents du XIX ${ }^{\mathrm{e}}$ siècle sont Etienne Geoffroy Saint-Hilaire (1772-1844) et son fils Isidore Geoffroy Saint-Hilaire (1805-1861). Un grand débat opposa en 1830 Etienne Geoffroy Saint-Hilaire au très protestant baron Georges Cuvier (1769-1832), partisan du fixisme, qui expliquait l'existence d'espèces «antédiluviennes » dont témoignaient des fossiles par de grandes catastrophes géologiques dont le Déluge serait l'archétype. Les historiens s'accordent à considérer que ce débat tourna à l'avantage du catastrophisme de Cuvier et que la plupart des naturalistes français ne se convertirent au transformisme qu'après la traduction en français de L'origine des espèces de Charles Darwin en $1862^{6}$.

Il faut croire que tous les contemporains ne partagèrent pas cet avis car Hippolyte Colins considérait dans Qu' est-ce que la science sociale? (1853-1854) que le transformisme était scientifiquement démontré. Dans Science sociale il affirme que la vie procède tout entière de la matière, qu'elle soit corporelle ou incorporelle, les forces étant naturellement purement matérielles ${ }^{7}$. "Notre globe a été primitivement à l'état igné. L'état igné est à la formation de l'univers ce que l'état aqueux est à la formation des organismes. L'humidité n'est apparue sur notre globe que plusieurs milliers d'années après son existence. Les végétaux et les animaux s'y sont développés successivement, en passant des plus simples aux plus composés. L'homme est le dernier des développements de l'organisme. La démonstration de ces faits scientifiques, il n'appartient qu'à l'ignorance: de la méconnaître. ${ }^{8}$ Aussi cette adhésion au transformisme est-elle un présupposé incontournable de sa «démonstration de l'immatérialité des âmes.» Comme Colins est décédé en 1859, l'année même de la parution de l'origine des espèces de Darwin, il est pour le moins hasardeux de le déclarer « antidarwiniste », comme le fait pourtant un auteur récent ${ }^{9}$.

\section{POSITION DU PROBLÈME PHILOSOPHIQUE}

Le problème philosophique fondamental pour Colins consiste à savoir si, par delà l'ordre physique régi par le déterminisme existe un ordre moral. Ce dernier ne saurait être fondé que sur l'âme. Colins identifie l'âme au sentiment que chacun a de sa propre existence, c'est-à-dire à la sensibilité réelle. Telle est son essence. Ce faisant il s'inscrit dans le prolongement de l'Ecole des idéologues,

Buican, Denis, L'évolution et les évolutionnismes, Que sais-je?. PUF, Paris, 1989, p. 45 ainsi que Grimoult, Cédric, Évolutionnisme et fixisme en France. Histoire d' un combat 1800-1882, CNRS Editions, 1998, p. 12.

6 Grimoult, Cédric, Ibidem, p. 139.

7 Colins vise par là l'énergie dont le concept scientifique ne fut dégagé qu'autour de 1850 par les physiciens anglais James Prescott Joule et William Thomson (alias Lord Kelvin). Dans l'ouvrage fondateur de la thermodynamique Réflexions sur le puissance motrice du feu et sur les machines propres à développer cette puissance qui date de 1824, Sadi Carnot parlait encore du calorique. Hippolyte Colins, Science sociale, tome V, p. 215.

9 Angenot, Marc, Colins et le socialisme rationnel, Les Presses universitaires de Montréal, Montréal, 1999, p. 165. 
eux-mêmes issus de la postérité de Condillac ${ }^{10}$. Toutefois, contrairement à la tradition dominante qui, dans la sensibilité, privilégie la perception des sens, il accorde la priorité au plaisir et à la peine, donc à la souffrance. Nous avons bien dit «sensibilité réelle», par opposition à la sensibilité apparente qui, si elle est purement matérielle, est illusoire. Certaines substances chimiques, certains appareils électriques sont dits sensibles, mais dans un sens figuré, par anthropomorphisme. Pour ce qui est de notre sensibilité, assurément réelle, tout le problème est de savoir si elle n'est qu'une fonction de notre organisme, auquel cas il n'y aurait tout simplement pas d'ordre moral distinct de l'ordre physique.

Colins ne met pas en doute que l'être humain ne puisse sentir et, a fortiori, penser sans son corps. Le problème est de savoir si ce dernier est le sujet de la sensation et de la pensée ou bien s'il n'en est que l'instrument, ce qui serait le cas si l'âme était démontrée immatérielle, donc non sujette au déterminisme non plus qu'au temps, autrement dit éternelle. Dans cette hypothèse, l'ordre moral constitué par les âmes dans leur union avec un organisme serait régi par la raison pure comme les mathématiques, ce en quoi Colins se rattache à une variante gnostique du rationalisme que l'on fait remonter à Pythagore. Dans cette hypothèse aussi, et nonobstant le transformisme, il n'y aurait pas «série continue des êtres » de l'amibe à l'homme et le «matérialisme prétendu scientifique ${ }^{11}$ serait controuvé.

\section{DÉMONSTRATION DE L'IMMATÉRIALITÉ DES ÂMES ${ }^{12}$}

Comme les âmes ne se manifestent qu'unies à un organisme matériel, la démonstration de leur immatérialité devra faire quelque emprunt à l'induction tout en recourant prioritairement à la déduction. En revanche, la science sociale qui en découlera procédera exclusivement par voie déductive. Comme tout rationaliste, Colins postule la véridicité de la raison et du raisonnement ${ }^{13}$.

L'axiome utilisé est celui de la sensibilité réelle de l'homme, dont la formulation est «je me sens exister», ou «je sens » ou encore plus synthétiquement «je». L'homme est un animal doté de sensibilité réelle. Mais celle-ci n'appartient pas nécessairement aux autres animaux dont la sensibilité mise en question sera dite apparente. Ce n'est qu'au terme de la démonstration que l'on saura de façon certaine si la sensibilité apparente des animaux est réelle ou illusoire.

10 Cf. François Picavet, Les idéologues. Essai sur l'histoire des idées et des théories scientifiques, philosophiques, religieuses, etc. en France depuis 1789, Félix Alcan, Paris 1891 et Sergio Moravia, Il pensiero degli Idéologues. Scienza e filosofia in Francia (1780-1815), «La Nuova Italia» Editrice, Firenze, 1974.

${ }_{11}$ Hippolyte Colins, Science sociale, tome I, p. 19, 177, et passim.

12 L'expression de «l'immatérialité de l'âme» avait été popularisée au XVIII" siècle par le fameux Treatise of Human Nature (1739-1740) de David Hume, dont une section était intitulée «Of the Immateriality of the Soul». Bien entendu, Colins prend le contrepied de l'illustre empiriste anglais.

${ }_{13}$ La démonstration en question fait l'objet des 260 première pages du tome V de Science sociale. Nous en avons entrepris l'analyse détaillée dans notre Introduction au socialisme rationnel de Colins, op. cit. pp. 289 à 367. Emmanuel Rens en a fait une lecture critique dans «Formulation logique de la démonstration colinsienne de l'immatérialité de l'âme » in Courants, Genève, 2000, p. 34,35 . 
Colins analyse un phénomène apparemment exceptionnel dans le règne animal dont il n'est pas déraisonnable de penser qu'il pourrait être la manifestation caractéristique de la sensibilité réelle, à savoir le langage qu'il appelle le verbe. Pour lui, le verbe est constitué par l'échange de signes convenus entre deux ou plusieurs sujets. Il ne s'agit donc pas du «langage articulé» comme le prétend un auteur récent ${ }^{14}$, le langage des sourds-muets constituant bien évidemment pour Colins un langage réel ${ }^{15}$.

Mais quelles sont les conditions requises pour qu'apparaisse le langage? Colins les réduit au nombre de trois:

a) une sensibilité réelle, qu'elle soit matérielle ou immatérielle,

b) un organisme doté de motilité et d'une mémoire matérielle centrale: le cerveau,

c) le non-isolement nécessaire, dans le sens déterministe du terme, des êtres supposés capables de développer le verbe.

Est-ce à dire que ces conditions étant réunies le langage se développera nécessairement? Hors l'hypothèse du don du langage par un Dieu créateur - que Colins tient pour absurde - il est bien évident que la communication linguistique est apparue spontanément entre les humains. D'ailleurs, observe Colins, bien que malaisée, l'expérimentation est ici possible et elle conclura comme le bon sens que le langage apparaît nécessairement lorsque sont réunies les conditions susénumérées.

Colins examine ensuite si le verbe se développe dans une catégorie beaucoup plus vaste d'êtres, à savoir ceux qui réunissent les conditions suivantes:

a) une sensibilité apparente, sans préjuger sa nature réelle ou illusoire,

b) un organisme doté de motilité et d'une mémoire matérielle centrale: le cerveau.

c) le non-isolement, dans le sens déterministe du terme, des êtres supposés capables de développer le verbe.

Autrement dit, les animaux possèdent-ils le langage, ou un langage, ou des langages? Pour répondre à cette question de façon aussi probante que possible, Colins décide de s'en tenir aux espèces dites supérieures, celles dont l'organisme est le plus proche de celui des hommes. Les différences entre certains grands singes et l'être humain sont en effet négligeables au regard du transformisme. Certes, les animaux dont il s'agit communiquent par des signes. Mais, aussi adaptés soient-ils, ces derniers ne sont nullement des signes convenus et ne constituent donc nullement des langages. La preuve c'est qu'on ne peut les traduire en langages humains ${ }^{16}$.

\footnotetext{
Angenot, Marc, op. cit., p. 50.

Hippolyte Colins, Science sociale, tome V, p. 227.

Nous sommes conscient que l'essor de l'éthologie intervenu depuis l'époque de Colins a conduit certains spécialistes à une position plus nuancée et que le mouvement de «libération animale» apparu depuis quelques décennies s'inscrit en faux contre cette conclusion. Nous nous réservons de les commenter de façon critique dans un ouvrage ultérieur.
} 
Vu que les conditions matérielles requises pour le développement du langage sont également réunies chez les hommes et chez les animaux qui ont été comparés, et vu que le langage s'est développé chez les uns à l'exclusion absolue de tous les autres, la sensibilité n'est réelle que chez les hommes à l'exclusion de tous les animaux, dont le nom même issu de anima, l'âme, est illusoire, de plus elle y est évidemment indépendante de la matière, donc immatérielle.

Par conséquent, la série des êtres comporte d'un côté des êtres purement matériels, de l'autre des êtres composés d'un organisme matériel ressortissant à ladite série et d'une immatérialité, de sorte que la série est rien moins que continue. CQFD

\section{DÉFINITION DE L'ORDRE MORAL}

Les événements tels qu'ils affectent l'homme ne sauraient constituer la condition nécessaires et suffisantes des souffrances et des jouissances, car ils procèdent tous plus ou moins de l'ordre physique alors que celles-ci relèvent exclusivement des sensibilités réelles, qui seules fondent la raison, la morale et le droit. Or, comme il est impossible de passer sans sophisme du fait au droit, de l'ordre physique à l'ordre moral, les souffrances et les jouissances, si même elles s'expliquent phénoménalement par des causes naturelles, ne se justifient métaphysiquement que par l'existence d'une loi suprême qui régit l'ordre moral comme le déterminisme régit l'ordre physique, mais qui transcende le déterminisme luimême comme l'âme incarnée transcende le corps.

Mais, objectera-t-on, comment la justification métaphysique de la condition humaine peut-elle se concilier avec l'explication physique des souffrances et des jouissances? D'ailleurs, une justification n'est-elle pas en définitive superfétatoire? Elle le serait évidemment si les hommes ne relevaient que de l'ordre physique et s'ils n'étaient inéluctablement, en leur qualité d'êtres raisonnables, amenés à s'interroger sur la raison de leur existence, le sens de leur vie, le but de leurs actions. Elle l'est d'ailleurs dans la pratique tant que la vérité n'est pas devenue absolument indispensable à la survie de l'humanité, car l'intelligence ne découvre que ce dont elle a impérieusement besoin. Aussi bien, si la société n'a jamais encore proclamé la vérité c'est qu'elle n'en a pas encore éprouvé la nécessité $^{17}$. Mais il n'en résulte pas que la raison métaphysique de la condition humaine soit superfétatoire pour l'avenir: «Mon travail est, à mes yeux, écrit Colins ${ }^{18}$, l'ouvrage le plus utile depuis l'origine sociale», car le temps arrive où l'humanité ne pourra survivre sans la connaissance de la vérité. Quant à la compatibilité de la justification métaphysique et de l'explication causale, il convient de remarquer qu'elles se situent sur deux plans différents. Pour Colins, dès que son point de départ métaphysique, l'immatérialité des âmes, a été identifié par la raison avec l'axiome «je me sens exister», la déduction nous livre en science sociale des vérités beaucoup plus sûres que l'induction dans les sciences naturelles, car les unes sont absolues comme les vérités mathématiques tandis que les autres ne sont

17 Hippolyte Colins, L'économie politique, source des révolutions et des utopies prétendues socialistes, Paris, 1857, tome II, p. 335.

18 Hippolyte Colins, La justice dans la science, hors l'Eglise et hors la Révolution, tome III, p. 210. 
qu'expérimentales donc relatives par essence et susceptibles de perfectionnements continus, ce qui est la marque même de l'imperfection.

«De l'immatérialité des âmes - écrit Colins - dérive nécessairement, la réalité de la LIBERTE, par conséquent la réalité de l'ordre moral, en absolue opposition avec l'ordre physique, où la liberté réelle ne peut exister; là où il ne peut y avoir que nécessité.

De l'immatérialité des âmes, de la réalité de leurs individualités, dérive nécessairement, la réalité de la raison: réalité qui est absurde dans toute autre hypothèse:

De la réalité de la raison, expression de l'ordre moral, résulte nécessairement aussi: que, la conformité à la raison, ou ce qui est rationnel, est l'expression de l'ordre moral.

De l'immatérialité des âmes et de la réalité de la raison, résulte, nécessairement encore, la responsabilité des actions commises conformément ou contrairement à la conscience, à la tendance de raison.

De cette responsabilité, il résulte, toujours nécessairement: que, toute action commise: contrairement à la conscience; ou conformément à la conscience et avec sacrifice de passion, avec sacrifice de tendance organique contraire à la raison; doit être punie ou récompensée.

Il en résulte encore, et toujours nécessairement: que toute action coupable ou méritoire, n'ayant point été punie ou récompensée dans une vie, doit nécessairement: être punie ou récompensée dans une ou plusieurs vies postérieures.

Il en résulte encore, et toujours nécessairement: que: toute souffrance ou jouissance non méritée dans la vie actuelle est, toujours nécessairement: la punition ou la récompense d'actions commises dans une vie antérieure.

Et, comme l'ordre moral n'est autre que l'ordre de justice, l'ordre de raison, il s'ensuit enfin, et toujours nécessairement: que, toute jouissance quelconque, toute souffrance quelconque est une récompense ou une expiation.

Cet ensemble de propositions se résume en disant:

L'ORDRE MORAL, C'EST L'HARMONIE ETERNELLE: ENTRE LA LIBERTE DES ACTIONS ; ET LA FATALITE DES EVENEMENTS.. ${ }^{19}$

\section{ECLAIRCISSEMENTS SUR LA MÉTEMPSY COSE COLINSIENNE}

Pour ce qui est de la métempsycose, Colins avait conscience de renouer avec une tradition vieille comme le monde représentée en Occident par les Druides et en Orient par l'hindouisme et le bouddhisme. Il convient toutefois de relever que sa doctrine se distingue tant de l'hindouisme que du bouddhisme par le fait qu'elle ne s'applique bien sûr qu'aux humains. Mais, dira-t-on, l'éternité des réincarnations est démentie par les données scientifiques qui déterminent avec suffisamment de précision l'époque à laquelle est apparu le genre humain. Certes, tel est le cas si l'on exclut l'existence d'autres humanités sur d'autres globes. Mais Colins

19 Hippolyte Colins, Qu' est-ce que la science sociale?, tome IV, pp. CLX et CLXI. Les italiques et les capitales sont de Colins. 
la postule au contraire, en raison de la vie limitée de notre Terre. Pourquoi n'y aurait-il pas des hommes sur Sirius, par exemple? Et qu'est-ce qui nous «empêche de parler avec les habitants de Sirius? La simple difficulté d'un perfectionnement optique. Qui donc oserait dire que ce perfectionnement est impossible ? ${ }^{20}$ Car, pour Colins, fondamentalement, l'homme est partout le même, en raison de l'immatérialité des âmes: «Tous les hommes, de tous les mondes existants et de tous les mondes possibles; de toutes les formes existantes, et de toutes les formes possibles; ont, non pas un même fonds d'idées; mais, le même fonds d'idées : parce qu'il n'y en a qu'un seul; et, qu'il ne peut y en avoir qu'un seul; ce fonds est la sensibilité.» ${ }^{21}$ Par conséquent, il nous «serait facile de parler avec les habitants de la lune; si même ils ressemblaient à des chauves-souris $»^{22}$.

L'une des particularités de la doctrine colinsienne de la métempsycose réside dans le fait qu'elle exclut tout souvenir d'une vie antérieure compte tenu du caractère essentiellement matériel de la mémoire. Autre originalité: en érigeant la morale en principe d'individuation, elle transpose dans un registre qui se veut rationnel l'un des dogmes les plus obscurs du christianisme, à savoir le péché originel.

$\mathrm{Au} \mathrm{XIX}^{\mathrm{e}}$ siècle les doctrines réincarnationistes connurent un regain de faveur en Occident en raison notamment de l'essor de l'orientalisme. Au demeurant, une philosophie fondée non point sur l'immortalité mais sur l'éternité des âmes et sur la rationalité de la morale se devait de déboucher sur une telle perspective.

\section{DE DIEU}

L'ordre moral, tel que défini plus haut, Colins l'appelle «éternelle raison» lorsqu'il parle logique, «éternelle justice», lorsqu'il parle éthique ou politique, ou «sanction religieuse» lorsqu'il parle métaphysique. A l'occasion, il l'appelle aussi $\operatorname{Logos}^{23}$ marquant ainsi la filiation qui le relie aux Stö̈ciens.

Le Dieu tout puissant, omniscient, créateur du ciel et de la Terre, n'est pour lui que l'image du despotisme, la négation de la liberté, de l'humanité. Prier Dieu pour lui demander telle ou telle faveur, intercéder auprès de lui, n'est-ce pas refaire le geste immémorial de l'esclave devant le maître, du serf devant le seigneur? Pour Colins, la prière, dans le sens habituel du terme, et l'intercession sous toutes ses formes, sont des insultes à l'éternelle justice, absolue, infinie, parfaite et immuable ${ }^{24}$. Si Dieu est absolu et infini, il est nécessairement impersonnel. La notion de personne implique celle d'organisme, donc elle évoque une existence temporelle, relative. Un Dieu personnel est une contradiction dans les termes.

\footnotetext{
$20 \quad$ Hippolyte Colins, Science sociale, tome V, p. 83.

21 Hippolyte Colins, Science sociale, tome V, p. 84.

22 Hippolyte Colins, Science sociale, tome V, p. 85.

${ }_{23}$ Hippolyte Colins, Science sociale, tome VI, p. 238-239.

24 Hippolyte Colins, La justice dans la science, hors l'Eglise et hors la Révolution, tome II, p. 355 et ss.
} 
Si donc Colins se dit volontiers athée, c'est parce qu'il ne croit pas en Dieu - la foi n'est-elle pas toujours l'aveu de l'ignorance? - mais, s'il n'y croit pas, c'est qu'il sait Dieu infini et absolu. Notre philosophe est donc athée par respect pour l'éternelle justice, c'est-à-dire pour le Logos, dont la majesté est si haute et si grande. Aussi bien rarement âme fut plus emplie que la sienne de l'amour du divin, comme en témoigne le passage suivant: «Et quel est donc l'homme de raison qui ait jamais proféré le blasphème horrible qu'il faut anéantir le sentiment? La raison doit le dominer, mais aussi longtemps qu'elle le domine, plus le sentiment se développe et plus l'homme est grand... Et pourquoi donc vouloir refuser au sentiment l'explosion d'amour de Dieu? Parce qu'elle est irrationnelle? S'adressant à l'anthropomorphe, oui: mais à l'éternelle justice, non. Sous l'empire de la raison, cette justice au contraire est incontestablement démontrée. Mais la justice éternelle, nous direz-vous encore, ce n'est pas un être réel, immatériel. Qui vous l'a dit? La raison n'en sait rien. Elle sait seulement qu'il est impossible et inutile de le savoir...» ${ }^{25}$

\section{APPRÉCIATION CRITIQUE}

On ne peut comprendre une pensée qu'en en mimant le mouvement, quitte à signaler les options qui apparaissent comme erronées. En l'occurrence, libre à chacun de considérer que la version colinsienne de l'argument ontologique pèche par ses prémisses ou par son recours illégitime au raisonnement par l'absurde.

Certes, Colins a eu quelques disciples de qualité, l'historien et révolutionnaire belge Louis De Potter (1786-1859), son fils le médecin Agathon De Potter (18271906), l'autodidacte français Elie Soubeyran (1863-1932) et, pendant quelque temps le naturaliste espagnol Ramón de la Sagra (1798-1871), pour n'en citer que quatre. Quant à son Ecole, elle ne vécut qu'environ cent ans comme le retrace l'ouvrage que nous lui avons consacrée ${ }^{26}$.

Parmi les obstacles qui s'opposent à l'accessibilité de cette doctrine figure son catastrophisme, c'est-à-dire sa conjecture que l'humanité ne manquerait pas de sombrer dans la violence généralisée si elle n'acceptait de se soumettre à la souveraineté de la raison et à la science sociale telle que découlant par voie logicodéductive de la démonstration de l'immatérialité des âmes. «Après dix, cent, mille, dix-mille révolutions, on est aussi avancé qu'au commencement: si le mal n'a pas été porté à l'excès suffisant... Le maniaque, on le saigne; la société se saigne elle-même. Le résultat, pour l'individu, est de reconnaître: qu'il est fou, le résultat pour la société, est de reconnaître: qu'elle est folle; et qu'elle a besoin de vérité. ${ }^{27} \mathrm{Ce}$ catastrophisme n'est pas sans évoquer celui du baron Cuvier, mais transposé de l'ordre physique à l'ordre politique et du passé dans l'avenir ! ${ }^{28}$ Pour l'avenir immédiat Colins prophétise l'avènement d'un ersatz de pouvoir spirituel,

25 Hippolyte Colins, Science sociale, tome VI, p. 105.

26 Ivo Rens et William Ossipow, Histoire d' un autre socialisme. L'Ecole colinsienne 1840-1940, La Baconnière, Neuchâtel, 1979.

27 Société nouvelle, sa nécessité, tome II, p. 59.

${ }^{28}$ Cf. à ce sujet Ivo Rens et Jacques Grinevald, «Réflexions sur le catastrophisme actuel», in Bernard Lescaze éd. Pour une histoire qualitative, Presse universitaires romandes, Genève, 1976, p. 283-321 ainsi que Science et anthropologie. Actes du Colloque international organisé à 
à savoir «la terreur de l'avenir $»^{29}$. «La terreur, pour que sa durée puisse se prolonger, doit nécessairement être vague. Un danger parfaitement connu cesse promptement d'être terrible; et il faudrait bien peu de jours pour dormir aussi tranquillement, sous l'épée de Damoclès, que sous la voûte des cieux. Aussi longtemps que le danger reste vague, au contraire, la terreur se prolonge et l'espérance n'a point d'accès.» ${ }^{30}$

Dans un ouvrage récent que nous avons déjà signalé, Marc Angenot résume la métaphysique «biscornue» (sic) de Colins par la formule lapidaire: "L'âme existe, donc Dieu n'existe pas ! $^{31}$ Malheureusement, à faire l'impasse sur la métaphysique de Colins on s'expose immanquablement à méconnaître des pans entiers de sa pensée économique et politique. Si Marc Angenot a compris que Colins préconisait la collectivisation du sol dans une économie de libre concurrence, il n'a pas saisi que cette dernière exigeait, toujours selon Colins, l'interdiction absolue des sociétés de capitaux: «Pour éviter la mort de l'humanité, la proscription d'associer les capitaux individuels, ou l'anéantissement de la féodalité financière, est devenue plus nécessaire encore: que la prohibition de l'assassinat $»^{32}$. Or, sans cette disposition, le socialisme colinsien demeure énigmatique ${ }^{33}$. C'est là une lacune regrettable car l'ouvrage en question se distingue par une tentative intéressante de reconstituer la doctrine de Colins à partir des écrits de ses disciples. Mais elle n'en est que plus représentative du conformisme culturel qui interdit aujourd'hui à la plupart des spécialistes des sciences sociales tout questionnement métaphysique.

Arbousse-en-Cévennes

l'occasion de deux-centième anniversaire de la naissance de Colins de Ham, Philosophie XI, ouvrage publié avec le concours du CNRS, Université de Toulouse-Le Mirail, 1984, passim.

29 Hippolyte Colins, Science sociale, tome II, p. 201, 206, et passim, mais surtout tome XVI, p. 147 à 208.

3o Hippolyte Colins, Science sociale, tome XVI, p. 167.

31 Angenot, Marc, Colins et le socialisme rationnel, op. cit., pp. 40 et ss.

32 Hippolyte Colins, De la justice dans la science, hors l' Eglise et hors la Révolution, tome I, p. 37. Au sujet de la genèse et de la place de ces sociétés dans l'ordre juridique. Cf. notre article «Sociétés de capitaux et idéologie capitaliste» in Bruno Schmidlin éd., Personne, société, nature, Editions universitaires, Fribourg, Suisse, 1996, p. 109-121.

33 A preuve: Marc Angenot affirme que «Colins est presque tout entier dans Saint-Simon», alors que Colins s'en démarque sur pratiquement tous les thèmes, et que Colins est «hostile au libre examen» alors que toute la philosophie de l'histoire de Colins est fondée sur l'avènement progressif mais définitif du libre examen ensuite de l'invention de l'imprimerie ! ( Angenot, Marc, op. cit., p. 167 et 168). Décidément Colins reste «Vox clamantis in deserto». 